\title{
Spatially-offset double-pulse laser-induced breakdown spectroscopy: A novel technique for analysis of thin deposited layers
}

\author{
D. Nishijima*, E.M. Hollmann, R.P. Doerner \\ Center for Energy Research, University of California at San Diego, \\ 9500 Gilman Dr., La Jolla, CA 92093-0417, USA
}

\begin{abstract}
A novel technique, spatially-offset double-pulse laser-induced breakdown spectroscopy (SODP-LIBS), is invented for analysis of thin layers. In this technique, two laser spots are spatially offset by a few $\mathrm{mm}$, while there is no spatial gap for the standard collinear DP-LIBS. It is demonstrated from analysis of thin W layers ( 30-250 nm thickness) deposited on Mo substrates at a reduced ambient pressure of $\sim 5 \times 10^{-3}$ Torr that (1) the W I signal intensity is enhanced with SODP-LIBS and (2) a clearer transition between W and Mo is obtained with SODP-LIBS, compared to the standard DP-LIBS.
\end{abstract}

Keywords: LIBS, spatially-offset double-pulse, thin deposited layer

\section{Introduction}

In fusion devices, plasma-facing materials (PFMs) are eroded by plasma bombardment, and eroded particles (atoms, molecules, dust, etc) are transported in the plasma and finally redeposit on other plasma-facing surfaces. Such thin deposited layers can significantly influence hydrogen isotope retention, sputtering yield of the substrate material, and substrate material properties. For instance, hydrogen isotopes can be retained more in a deposited layer than in a bulk material, which may lead to a serious safety concern [1]. Another example is alloy formation between a deposited layer and the bulk

\footnotetext{
* Corresponding author

Email address: dnishijima@eng.ucsd.edu (D. Nishijima)
} 
material. Such alloys may have a much lower melting temperature than that of the bulk material, e.g. in ITER, the first wall material, beryllium (Be), deposition on the divertor made of tungsten (W) tiles can lead to the formation of Be-W alloys with lower melting temperatures $\left(\sim 2520 \mathrm{~K}\right.$ for $\mathrm{Be}_{2} \mathrm{~W}$, $\sim 1780 \mathrm{~K}$ for $\mathrm{Be}_{12} \mathrm{~W}$, and $\sim 1600 \mathrm{~K}$ for $\left.\mathrm{Be}_{22} \mathrm{~W}\right)$ than the bulk W (3695 K) [2]. Thus, in situ monitoring of deposited layers in fusion devices is thought to be quite beneficial to promoting the understanding of complicated plasmamaterial interactions. Furthermore, in the future fusion devices with tritium, removal of PFM tiles for post-mortem analysis will be more restricted [3]. In this sense, the development of in situ diagnostic techniques of PFM surfaces is an urgent issue.

Laser-induced breakdown spectroscopy (LIBS) $[4,5,6,7]$ is one of the candidate techniques for in situ analysis of PFM surfaces in fusion devices $[3,8]$, because of its capability of remote-sensing. A possible issue of LIBS in fusion devices is that the signal intensity may be weak, as the ambient pressure is low or may not be optimized, and light collection optics are situated far from PFM unless a robotic arm is used to locate them close to PFM. To overcome this issue, double-pulse (DP) LIBS [7, 9] may be applied, which has been generally used for bulk material analysis around atmospheric pressure. A thick deposited layer ( $\sim 3 \mu \mathrm{m}$ thickness) has been analyzed with DP-LIBS, and a signal enhancement of a factor of $\sim 2$ was obtained [10]. However, the applicability of DP-LIBS for thinner layers remains unclear. Note that, while there are a few DP-LIBS configurations, the collinear configuration is thought to be most appropriate in fusion devices from the geometrical point of view.

In this paper, we show that the standard collinear DP-LIBS cannot be used for thin layers, the layer thickness of which is thinner than or comparable to the ablation rate, unlike thicker layers and bulk materials. Then, we propose a novel technique, spatially-offset double-pulse LIBS (SODP-LIBS), for analysis of thin layers. This technique is the same as the collinear DPLIBS, except that two laser spots on a sample are spatially offset by a few $\mathrm{mm}$. The advantage of SODP-LIBS is demonstrated with thin $\mathrm{W}$ layers ( $\sim 30$ $\mathrm{nm}, 60 \mathrm{~nm}$, and $250 \mathrm{~nm}$ ) deposited on molybdenum (Mo) substrates.

\section{Experimental setup}

A schematic layout of the experimental setup is depicted in figure 1 (a). Two Q-switched Nd:YAG lasers (Continuum Surelite III-10 and II-10) were used at the output laser wavelength of $1064 \mathrm{~nm}$. The pulse width is nom- 
inally $\sim 5$ ns for III-10 and $\sim 6$ ns for II-10. The laser beam spatial profile is close to Gaussian. Since the polarization of both lasers is horizontal, the polarization of the second laser (II-10) is rotated to be vertical with a halfwave plate (HWP). A polarizing beam combiner (PBC) makes two laser beam paths collinear. For SODP-LIBS, the first and second beam paths are slightly shifted by adjusting mirrors to make a spatial separation, $\Delta L_{12}$, on the target surface. The laser pulses are focused with a plano-convex lens (focal length $=250 \mathrm{~mm}$ ) onto a target located inside a vacuum chamber. The laser spot diameter is around $800 \mu \mathrm{m}$, observed with a laser confocal microscope (Olympus LEXT OLS4100). The laser energy, $E_{\mathrm{L}}$, is controlled by varying the Q-switch delay time. In DP- and SODP-LIBS, the first $\left(E_{\mathrm{L} 1}\right)$ and second $\left(E_{\mathrm{L} 2}\right)$ pulses were set to have the same energy, i.e. $E_{\mathrm{L} 1}=E_{\mathrm{L} 2}=$ $115 \mathrm{~mJ}$, while single-pulse (SP) LIBS was done with $E_{\mathrm{L}}=115 \mathrm{~mJ}$ and 230 $\mathrm{mJ}$. The base pressure of the chamber is around $5 \times 10^{-3}$ Torr, pumped down only with a rotary pump. The experiments were carried out at this pressure without injecting gas.

Plasma light emission was collected with a lens (focal length $=100 \mathrm{~mm}$, diameter $=50 \mathrm{~mm}$ ), and delivered through an optical fiber (core diameter $=$ $600 \mu \mathrm{m}, \mathrm{NA}=0.39$ ) to the entrance slit of an Echelle spectrometer (Andor ME5000) equipped with an ICCD camera (Andor iStar DH334T). The distance between the lens and the target is around $200 \mathrm{~mm}$, and the diameter of the observation spot on the target is around $23 \mathrm{~mm}$. Another ICCD camera (Princeton Instruments PI-MAX) was used to take plasma images, looking from the side of the target. The time sequence is shown in figure 1 (b), where $\Delta t_{12}$ is the interpulse separation time. In the experiments presented here, the ICCD delay time, $t_{\text {delay }}$, and the ICCD gate width, $t_{\text {width }}$, were set to $0.13 \mu \mathrm{s}$ and $10 \mu \mathrm{s}$, respectively. With this $t_{\text {delay }}$, the strong continuum emission is excluded. The whole system was synchronized with two digital delay generators (SRS DG535). Note that $t_{\text {delay }}$ is relative to the second pulse. Following reference [7], a bundle of the 1st and 2nd pulses is called a burst hereafter, which is sometimes used even for a laser pulse of SP-LIBS. Three spectra were typically collected for each condition, and were averaged for better accuracy.

Thin $\mathrm{W}$ layers were created on mirror-polished Mo substrates in a magnetron sputtering device. A small area of the substrate was covered with an aluminum foil, so that the step height between the covered and uncovered areas becomes the $\mathrm{W}$ layer thickness, $\Delta W$, and was measured with the laser confocal microscope. 

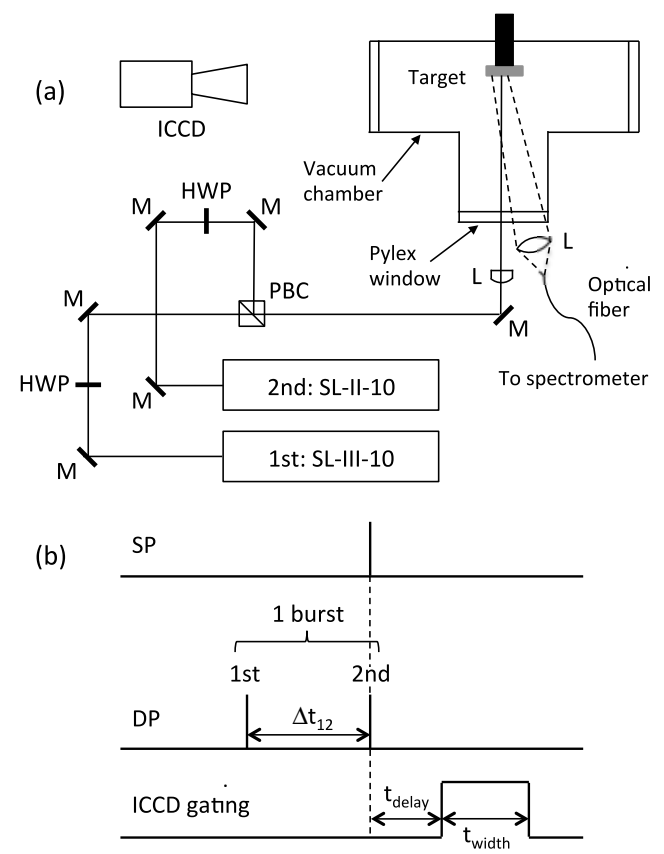

Figure 1: (a) Schematic of the experimental setup and (b) the time sequence. The timing of two lasers and ICCD cameras is controlled with two digital delay generators. M: mirror, HWP: half-wave plate, PBC: polarizing beam combiner, L: lens.

\section{Experimental results and discussion}

In figure 2, W I $429.4 \mathrm{~nm}$ line emission sepctra are compared between SP, DP- $\left(\Delta t_{12}=5.32 \mu \mathrm{s}\right)$, and SODP-LIBS $\left(\Delta t_{12}=5.32 \mu \mathrm{s}, \Delta L_{12} \sim 1.5 \mathrm{~mm}\right)$. The target is a thin $\mathrm{W}$ layer $(\Delta W \sim 60 \mathrm{~nm})$ deposited on a Mo substrate. The spectra were take from the 1st burst. As is clearly seen, SODP-LIBS generates the highest intensity compared to the others. In addition, the signal to background, $S / B$, ratio of SODP-LIBS $(\sim 7.1)$ is around 2.5 times higher than the others.

Depth profiling of thin $\mathrm{W}$ layers on Mo substrates with $\Delta W \sim 30 \mathrm{~nm}$, $60 \mathrm{~nm}$, and $250 \mathrm{~nm}$ was also performed. Figure 3 shows the intensity of the W I $429.4 \mathrm{~nm}$ and Mo I $550.6 \mathrm{~nm}$ lines as a function of the burst number, where $\Delta t_{12}$ was set to $5.32 \mu \mathrm{s}$, and $\Delta L_{12}$ was slightly different $(\sim 1.3-1.7$ $\mathrm{mm}$ ) for each layer. First of all, SODP-LIBS yields the highest W I signal at the 1st burst among the others in all the layers. Compared to SP-LIBS at $E_{\mathrm{L}}=115 \mathrm{~mJ}$, the signal is enhanced by a factor of $\sim 2.0,2.7$, and 3.4 for 


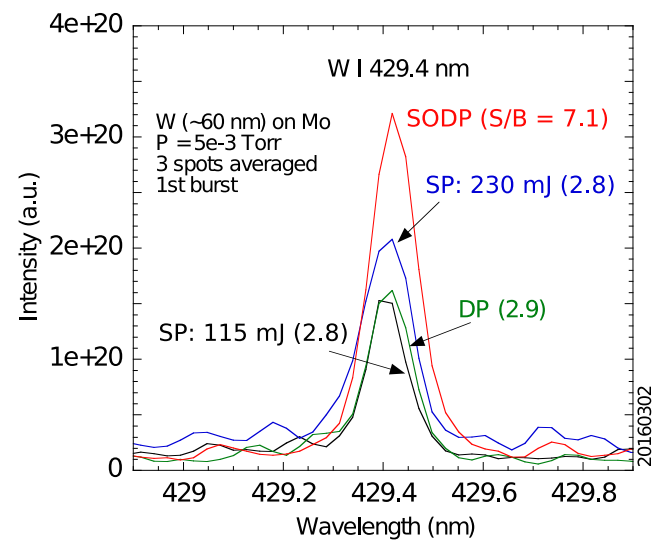

Figure 2: W I $429.4 \mathrm{~nm}$ line excited by SP-LIBS, DP-LIBS $\left(\Delta t_{12}=5.32 \mu \mathrm{s}\right)$, and SODPLIBS $\left(\Delta t_{12}=5.32 \mu \mathrm{s}, \Delta L_{12} \sim 1.5 \mathrm{~mm}\right)$. The target is a thin $\mathrm{W}(\Delta W \sim 60 \mathrm{~nm})$ layer on a Mo substrate. The spectra were taken from the 1st burst. The $S / B$ ratio for each method is shown in parentheses.

$\Delta W \sim 30 \mathrm{~nm}, 60 \mathrm{~nm}$, and $250 \mathrm{~nm}$, respectively. DP-LIBS does not enhance the W I signal compared to SP-LIBS for the layers with $\Delta W \sim 30 \mathrm{~nm}$ and $60 \mathrm{~nm}$, while a factor of $\sim 2$ enhancement is seen at $\Delta W \sim 250 \mathrm{~nm}$.

The transition between the $\mathrm{W}$ layer and the Mo substrate is clearly observed with SODP-LIBS for all the layers, i.e. the higher W I signal and the lower Mo I signal at the 1st burst, and the very weak W I signal and the flat Mo I signal afterwards. Since the average ablation rate was measured to be $\sim 250 \mathrm{~nm} /$ pulse with a bulk W sample, the weak Mo I line is already detected at the 1st burst for the thinner layers with $\Delta W \sim 30 \mathrm{~nm}$ and 60 nm. On the other hand, DP-LIBS exhibits a flat depth profile of the Mo I signal for all the layers. This is because (1) the 1st pulse of the 1st burst removes the $\mathrm{W}$ layer, and thus (2) the 2 nd pulse of the 1st burst ablates the Mo substrate on the same spot. As mentioned above, this fatal weak point of DP-LIBS is overcome by SODP-LIBS, as the 2nd pulse of the 1st burst hits a fresh $\mathrm{W}$ layer surface. It should be noted that this flat Mo I signal as well as the non-enhanced W I signal measured by DP-LIBS indicate that the effect of plasma reheating by the 2nd pulse is negligible.

Concerning SP-LIBS, $E_{\mathrm{L}}=230 \mathrm{~mJ}$ is too high to probe the thin layer with $\Delta W \sim 30 \mathrm{~nm}$, since the depth profile of the Mo I signal is flat. At $E_{\mathrm{L}}$ $=115 \mathrm{~mJ}$, the Mo I signal intensity and profile look very similar to SODP- 

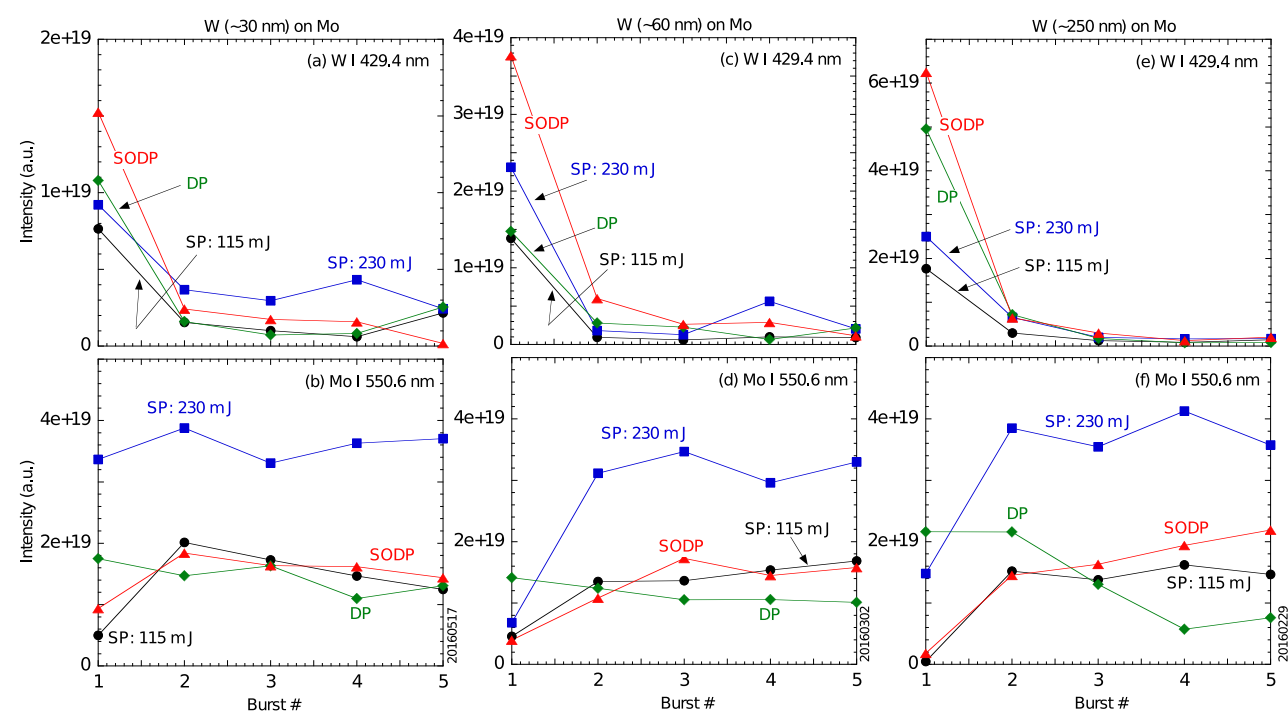

Figure 3: Depth profiling of thin $\mathrm{W}$ layers on Mo substrates using SP-LIBS, DP-LIBS $\left(\Delta t_{12}=5.32 \mu \mathrm{s}\right)$, and SODP-LIBS $\left(\Delta t_{12}=5.32 \mu \mathrm{s}\right)$. (a) (b) $\Delta W \sim 30 \mathrm{~nm}$ and $\Delta L_{12} \sim$ $1.3 \mathrm{~mm}$, (c) (d) $\Delta W \sim 60 \mathrm{~nm}$ and $\Delta L_{12} \sim 1.5 \mathrm{~mm}$, (e) (f) $\Delta W \sim 250 \mathrm{~nm}$ and $\Delta L_{12} \sim 1.7$ mm. (a) (c) (e) W I $429.4 \mathrm{~nm}$ line intensity, and (b) (d) (f) Mo I $550.6 \mathrm{~nm}$ line intensity.

LIBS. This indicates that SODP-LIBS does not lead to a signal enhancement of a bulk material in this condition, i.e. a low ambient pressure and $\Delta t_{12}=$ $5.32 \mu$ s. Later, we will show a signal enhancement of a bulk W sample with a shorter $\Delta t_{12}$.

In the depth profiling presented in figure $3, \Delta L_{12}$ is slightly different, ranging from $\sim 1.3-1.7 \mathrm{~mm}$. To explore the sensitivity of the $\mathrm{W}$ I signal intensity to $\Delta L_{12}$, the W I signal was measured with SODP-LIBS while varying $\Delta L_{12}$, where a bulk W sample was used. Figure 4 demonstrates that the $\Delta L_{12}$ dependence of the $\mathrm{W}$ I intensity is very weak in the range of 1-4 $\mathrm{mm}$. This weak $\Delta L_{12}$ dependence is another beneficial feature of SODPLIBS in a practical use, as the precise alignment of two laser spots is not required.

To pursue a further signal enhancement, $\Delta t_{12}$ was varied. With a bulk W sample, the $\Delta t_{12}$ dependence of the W I $429.4 \mathrm{~nm}$ line intensity was investigated using both DP- and SODP-LIBS. It is found from figure 5 (a) that the $\Delta t_{12}$ dependence for both techniques is very similar, and the intensity peaks at $\Delta t_{12} \sim 1 \mu$ s, i.e. $\sim 2$ times enhancement compared to $\Delta t_{12} \sim 5.32 \mu$ s. Note 


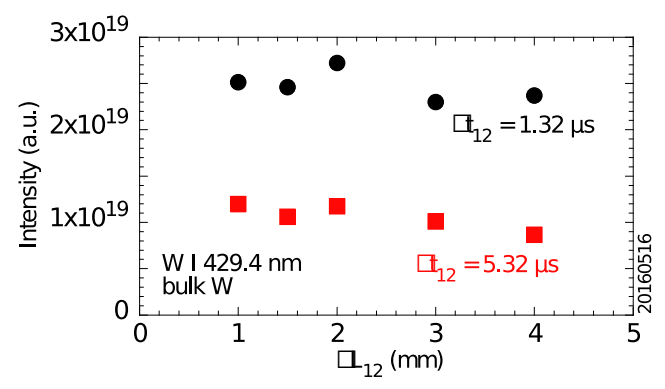

Figure 4: $\Delta L_{12}$ dependence of the W I $429.4 \mathrm{~nm}$ line intensity taken with SODP-LIBS $\left(\Delta t_{12}=1.32 \mu \mathrm{s}\right.$ (circles) and $\Delta t_{12}=5.32 \mu \mathrm{s}$ (squares)) on a bulk W sample.

that the intensity for DP-LIBS is slightly higher than that for SODP-LIBS, the reason for which is discussed later.

A thin $\mathrm{W}$ layer $(\Delta W \sim 30 \mathrm{~nm})$ was, therefore, re-analyzed using SODPLIBS as well as DP-LIBS with $\Delta t_{12}=1.32 \mu \mathrm{s}$. As seen in figure $5(\mathrm{~b})$, the $\mathrm{W}$ I signal of SODP-LIBS with $\Delta t_{12}=1.32 \mu \mathrm{s}$ is enhanced by a factor of $\sim 2.5$ compared to $\Delta t_{12}=5.32 \mu \mathrm{s}$. This means $\sim 5$ times enhancement against SP-LIBS at $E_{\mathrm{L}}=115 \mathrm{~mJ}$. On the other hand, the signal increase $(\sim 50 \%)$ is much smaller for DP-LIBS unlike a bulk sample.

The following mechanism can be considered for the signal enhancement of thin W layers with SODP-LIBS. The 1st laser pulse ablates a W layer, and produces a $\mathrm{W}$ plasma. When the 2 nd laser pulse hits a fresh nearby spot of the $\mathrm{W}$ layer, $\mathrm{W}$ atoms ablated by the 1st pulse still exist in the volume in front of the surface. Some of those residual W atoms are still populated in excited states, and emit light. The fraction of such light-emitting $\mathrm{W}$ atoms is higher at shorter $\Delta t_{12}$, as shown with circles in figure 5 (a). The data was taken only with the 1st laser pulse by blocking the 2nd pulse. For instance, the $\mathrm{W}$ I intensity from light-emitting $\mathrm{W}$ atoms of the 1st plasma is around $20 \%$ of the observed intensity at $\Delta t_{12}=1.32 \mu \mathrm{s}$, while it is negligibly small at $5.32 \mu \mathrm{s}$. In addition, other $\mathrm{W}$ atoms in the ground and metastable states are re-excited by the 2 nd plasma, leading to light emission. Of course, W atoms ablated by the 2nd pulse contribute to the observed intensity. In this way, SODP-LIBS can enhance the signal from a thin W layer.

To support this hypothesis, two-dimensional plasma images were taken with an ICCD camera, as the plasma volume (or the light emitting volume) of SODP-LIBS is expected to be larger than SP-LIBS. A narrow bandpass 

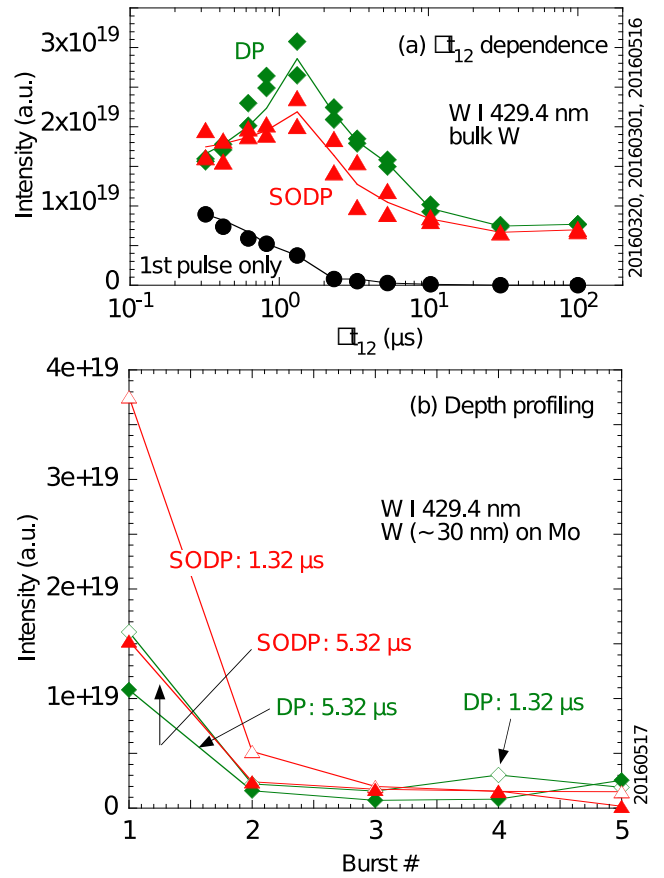

Figure 5: (a) $\Delta t_{12}$ dependence of the W I $429.4 \mathrm{~nm}$ line intensity taken with DP-LIBS and SODP-LIBS $\left(\Delta L_{12} \sim 1.6 \mathrm{~mm}\right)$ on a bulk $\mathrm{W}$ sample. The intensity taken only with the 1st pulse is also shown. The lines are drawn only for the guide of eyes. (b) Depth profiling of a thin W layer $(\Delta W \sim 30 \mathrm{~nm})$ on a Mo substrate using DP- (diamonds) and SODP-LIBS (triangles) with $\Delta t_{12}=1.32 \mu \mathrm{s}$ (open symbols) and $5.32 \mu \mathrm{s}$ (closed symbols). The data at $\Delta t_{12}=5.32 \mu \mathrm{s}$ is the same as figure $3 . \Delta L_{12} \sim 1.3 \mathrm{~mm}$.

filter was placed in front of the camera lens, and the central wavelength of the filter, $\lambda_{\mathrm{c}}$, is $430.2 \mathrm{~nm}$, and its FWHM (full width at half maximum) is 3 $\mathrm{nm}$. Thus, two W I lines at $429.4 \mathrm{~nm}$ and $430.2 \mathrm{~nm}$ pass through the filter. Figure 6 shows false color images of the W I emission produced by the 1st burst of SP-, DP- and SODP-LIBS on a thin W layer with $\Delta W \sim 60 \mathrm{~nm}$ deposited on a Mo substrate. For SODP-LIBS, $\Delta L_{12}$ is $\sim 1.5 \mathrm{~mm}$, and the two laser spots are arranged to be parallel to the line-of-sight of the ICCD camera. Since the intensity was weak and somewhat noisy, the raw data was smoothed out with a boxcar average of the width of 5 CCD pixels. As can be seen, SODP-LIBS produces a larger plasma volume than the others, resulting in the signal enhancement.

It should be noted that this enhancement mechanism is common to DP- 

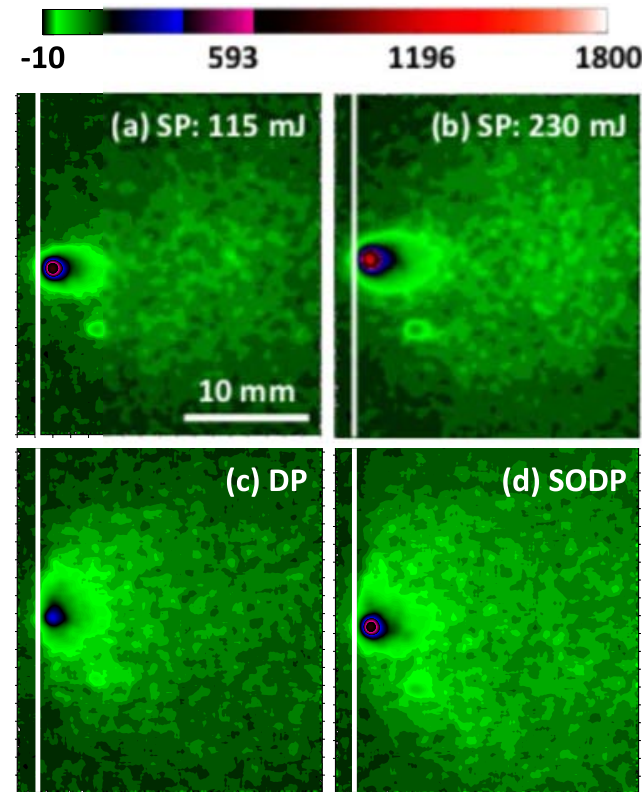

Figure 6: False color images of plasmas produced by the 1st burst of (a) SP-LIBS with $E_{\mathrm{L}}=115 \mathrm{~mJ}$, (b) SP-LIBS with $E_{\mathrm{L}}=230 \mathrm{~mJ}$, (c) DP-LIBS $\left(\Delta t_{12}=5.32 \mu \mathrm{s}\right)$, and (d) SODP-LIBS $\left(\Delta t_{12}=5.32 \mu \mathrm{s}, \Delta L_{12} \sim 1.5 \mathrm{~mm}\right)$. The target is a thin $\mathrm{W}$ layer $(\Delta W \sim 60$ $\mathrm{nm})$ on a Mo substrate. The images were taken through a narrow bandpass filter $\left(\lambda_{\mathrm{c}}=\right.$ $430.2 \mathrm{~nm}, \mathrm{FWHM}=3 \mathrm{~nm})$. Raw data was smoothed with a boxcar average of the width of 5 pixels. The vertical lines indicate the target position.

LIBS for a bulk $\mathrm{W}$ sample, since the 2nd pulse also irradiates the $\mathrm{W}$ surface. That is why the plasma image induced by DP-LIBS is similar to SODPLIBS, while the W I intensity is weaker with DP-LIBS because the 2nd laser pulse interacts with the substrate and not the thin W layer. For DP-LIBS on bulk W, another process needs to be considered. When the 2nd pulse hits the same spot as the 1st pulse, the temperature at the spot is expected to be higher than room temperature, depending on $\Delta t_{12}$. In fact, a larger crater volume for DP-LIBS is observed compared to SP-LIBS. This increased ablation may be responsible for the higher intensity than SODP-LIBS for bulk $\mathrm{W}$, as observed in figure 5 (a). 


\section{Conclusion}

In conclusion, we demonstrated that the performance of the newly developed SODP-LIBS technique is superior to SP-LIBS and the standard collinear DP-LIBS for thin layer analyses. Re-excitation of residual $\mathrm{W}$ atoms in the volume by the 2nd plasma is considered to be the key mechanism of the signal enhancement. SODP-LIBS will facilitate in situ analyses of thin layers deposited on PFM surfaces in fusion devices, which will aid in understanding the physics of plasma-surface interactions. Moreover, SODP-LIBS will be used to measure any thin films in industry applications. It should be, however, noted that SODP-LIBS cannot be applied for quantitative analyses of thin layers, the composition of which significantly changes in a few $\mathrm{mm}$, since there is a spatial offset of a few $\mathrm{mm}$ or less between two laser spots.

\section{Acknowledgments}

We would like to thank Mr. C. Jacobsen for preparing W coatings on Mo substrates. This work is supported by the U.S. Department of Energy Grant No. DE-FG02-07ER54912.

\section{References}

[1] J. Roth et al., J. Nucl. Mater. 390-391 (2009) 1.

[2] M.J. Baldwin, R.P. Doerner, D. Nishijima, D. Buchenauer, W.M. Clift, R.A. Causey and K. Schmid, J. Nucl. Mater. 363-365 (2007) 1179.

[3] V. Philipps et al, Nucl. Fusion 53 (2013) 093002.

[4] A. Miziolek, V. Palleschi and I. Schechter, Laser-Induced Breakdown Spectroscopy (LIBS): Fundamentals and Applications (Cambridge: Cambridge University Press) (2006).

[5] D.W. Hahn and N. Omenetto, Applied Spectroscopy 64 (2010) 335A.

[6] D.W. Hahn and N. Omenetto, Applied Spectroscopy 66 (2012) 347.

[7] R. Noll, Laser-Induced Breakdown Spectroscopy (Berlin Heidelberg: Springer) (2012). 
[8] L. Mercadier, A. Semerok, P.A. Kizub, A.V. Leontyev, J. Hermann, C. Grisolia, P.-Y. Thro, J. Nucl. Mater. 414 (2011) 485.

[9] J. Pender et al, Laser-induced breakdown spectroscopy using sequential laser pulses, in Laser-Induced Breakdown Spectroscopy (LIBS): Fundamentals and Applications, chapter 15, ed. A. Miziolek, V. Palleschi and I. Schechter, (Cambridge: Cambridge University Press) (2006).

[10] S. Almaviva, L. Caneve, F. Colao, P. Gasior, M. Kubkowska, M. Lepek, G. Maddaluno, Fus. Eng. Des. 96-97 (2015) 848. 


\section{Graphical abstract}
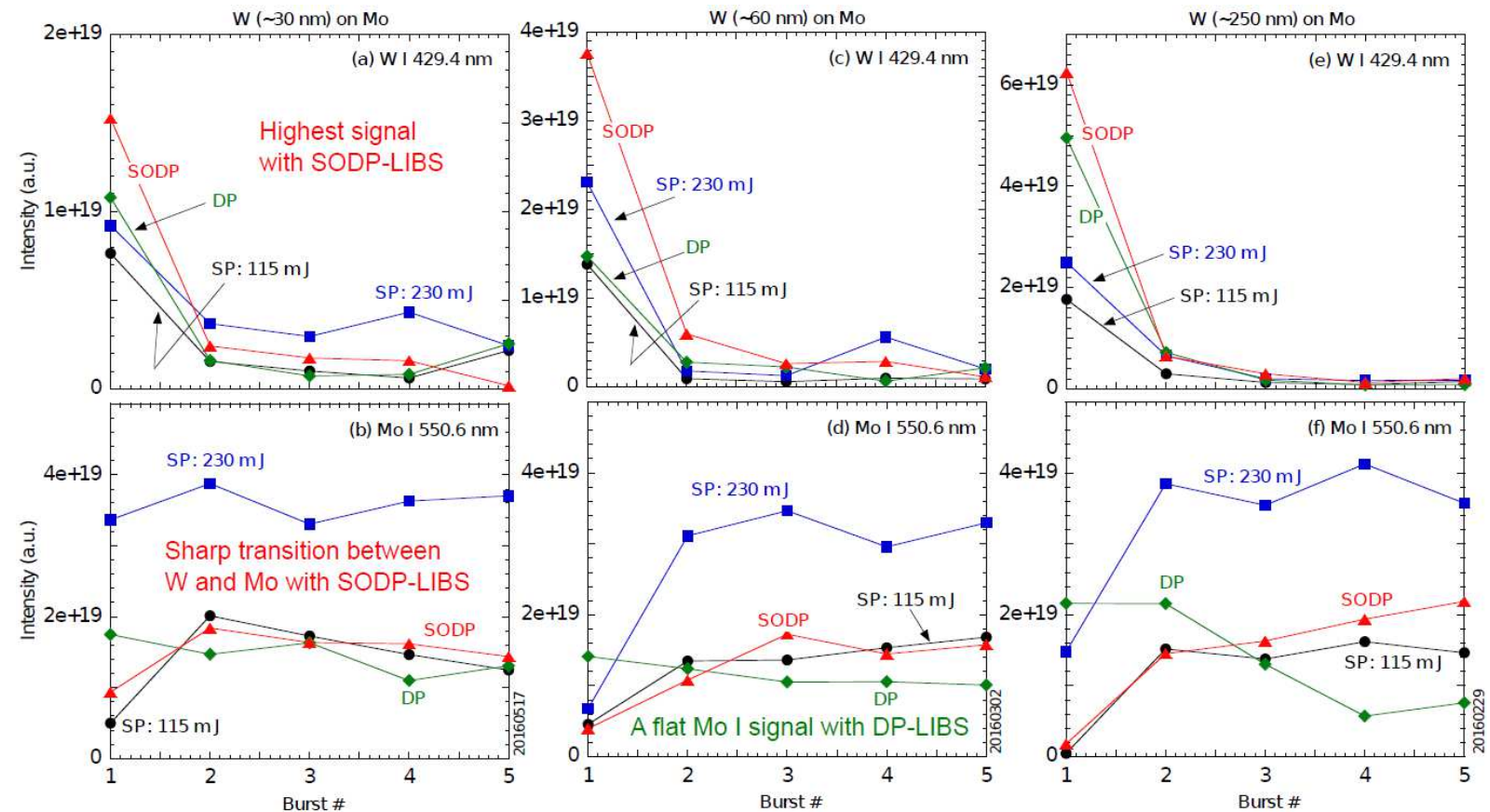